\title{
Classification of Large-Scale High-Resolution SAR Images With Deep Transfer Learning
}

\author{
Zhongling Huang $\left.{ }^{(}\right)$, Corneliu Octavian Dumitru ${ }^{\circledR}$, Zongxu Pan ${ }^{\circledR}$, Member, IEEE, \\ Bin Lei, and Mihai Datcu, Fellow, IEEE
}

\begin{abstract}
The classification of large-scale high-resolution synthetic aperture radar (SAR) land cover images acquired by satellites is a challenging task, facing several difficulties such as semantic annotation with expertise, changing data characteristics due to varying imaging parameters or regional target area differences, and complex scattering mechanisms being different from optical imaging. Given a large-scale SAR land cover data set collected from TerraSAR-X images with a hierarchical three-level annotation of 150 categories and comprising more than 100000 patches, three main challenges in automatically interpreting SAR images of highly imbalanced classes, geographic diversity, and label noise are addressed. In this letter, a deep transfer learning method is proposed based on a similarly annotated optical land cover data set (NWPU-RESISC45). Besides, a top-2 smooth loss function with cost-sensitive parameters was introduced to tackle the label noise and imbalanced classes' problems. The proposed method shows high efficiency in transferring information from a similarly annotated remote sensing data set, a robust performance on highly imbalanced classes, and is alleviating the overfitting problem caused by label noise. What is more, the learned deep model has a good generalization for other SAR-specific tasks, such as MSTAR target recognition with a state-of-the-art classification accuracy of $99.46 \%$.
\end{abstract}

Index Terms-High-resolution (HR) synthetic aperture radar (SAR) images, label noise, land cover classification, TerraSAR-X (TSX), transfer learning.

\section{INTRODUCTION}

$\mathbf{S}$ YNTHETIC aperture radar (SAR) land use and land cover (LULC) classification is an important step in SAR

Manuscript received December 18, 2018; revised April 1, 2019, September 1, 2019, and December 11, 2019; accepted January 7, 2020. Date of publication January 22, 2020; date of current version December 24, 2020. This work was supported by the National Natural Science Foundation of China under Grant 61701478, and the University of Chinese Academy of Sciences (UCAS) Joint Ph.D. Training Program scholarship. (Corresponding author: Mihai Datcu.)

Zhongling Huang is with the Aerospace Information Research Institute, Chinese Academy of Sciences, Beijing 100094, China, also with the School of Electronic, Electrical and Communication Engineering, University of Chinese Academy of Sciences, Beijing 101408, China, also with the Key Laboratory of Technology in Geo-Spatial Information Processing and Application System, Chinese Academy of Sciences, Beijing 100094, China, and also with the EO Data Science Department, German Aerospace Center (DLR), 82234 Wessling, Germany (e-mail: huangzhongling15@mails.ucas.ac.cn).

Corneliu Octavian Dumitru and Mihai Datcu are with the EO Data Science Department, German Aerospace Center (DLR), 82234 Wessling, Germany (e-mail: corneliu.dumitru@dlr.de; mihai.datcu@dlr.de).

Zongxu Pan and Bin Lei are with the Aerospace Information Research Institute, Chinese Academy of Sciences, Beijing 100094, China, also with the School of Electronic, Electrical and Communication Engineering, University of Chinese Academy of Sciences, Beijing 101408, China, and also with the Key Laboratory of Technology in Geo-Spatial Information Processing and Application System, Chinese Academy of Sciences, Beijing 100094, China.

Color versions of one or more of the figures in this letter are available online at https://ieeexplore.ieee.org.

Digital Object Identifier 10.1109/LGRS.2020.2965558 image interpretation. Recognizing different land covers in SAR images requires expertise in interpreting the complex scattering characteristics under various circumstances, such as different wavelengths, incidence angles, and target areas, which makes the annotation of large-scale SAR data sets timeconsuming. With the development of deep learning algorithms, hierarchical features can be extracted automatically. Unsupervised learning approaches, such as deep belief networks (DBNs) [1] and autoencoders (AEs) [2], [3], are widely applied to SAR images for classification. With more detailed texture information, high-resolution (HR) PolSAR images are used to learn spatial and polarimetric features jointly via deep convolutional neural networks (DCNNs) for supervised classification, which achieves state-of-the-art performance [4].

However, many SAR LULC classification algorithms mainly focus on specific tasks that may not generalize well on other SAR tasks, for example, classifying vegetation and agricultural land covers in farmland areas [5] or training an urban-specific network with two scene images in urban areas [6]. Although a big volume of SAR data all over the world is acquired every day, it is challenging to automatically interpret land covers in SAR images with such diversity and to obtain a well-generalized model for SAR image understanding.

In this letter, a global data set of multilevel annotated HR SAR images [7] has been experimented. These SAR images vary in geographic locations, incidence angles, radiometric calibration factors, and ascending or descending satellite orbit branches. With the difficulties of highly imbalanced categories, geographic diversity, and noisy annotations, our contributions are as follows.

1) A very deep residual network with a transitive transfer learning method from natural images to remote sensing and then to SAR data is used to understand a large amount of land cover SAR images from all over the world.

2) A cost-sensitive top- 2 smooth combined loss function with cross-entropy loss is proposed to alleviate the imbalanced problem and model bias to mislabeled training samples.

3) The proposed network is proven to have a good generalization of other SAR data sets and tasks.

In the following, we will first introduce the TerraSAR-X (TSX) annotated land cover data set in Section II. Our transfer learning-based method and the experimental results will be presented in Sections III and IV, followed by a conclusion in Section V. 


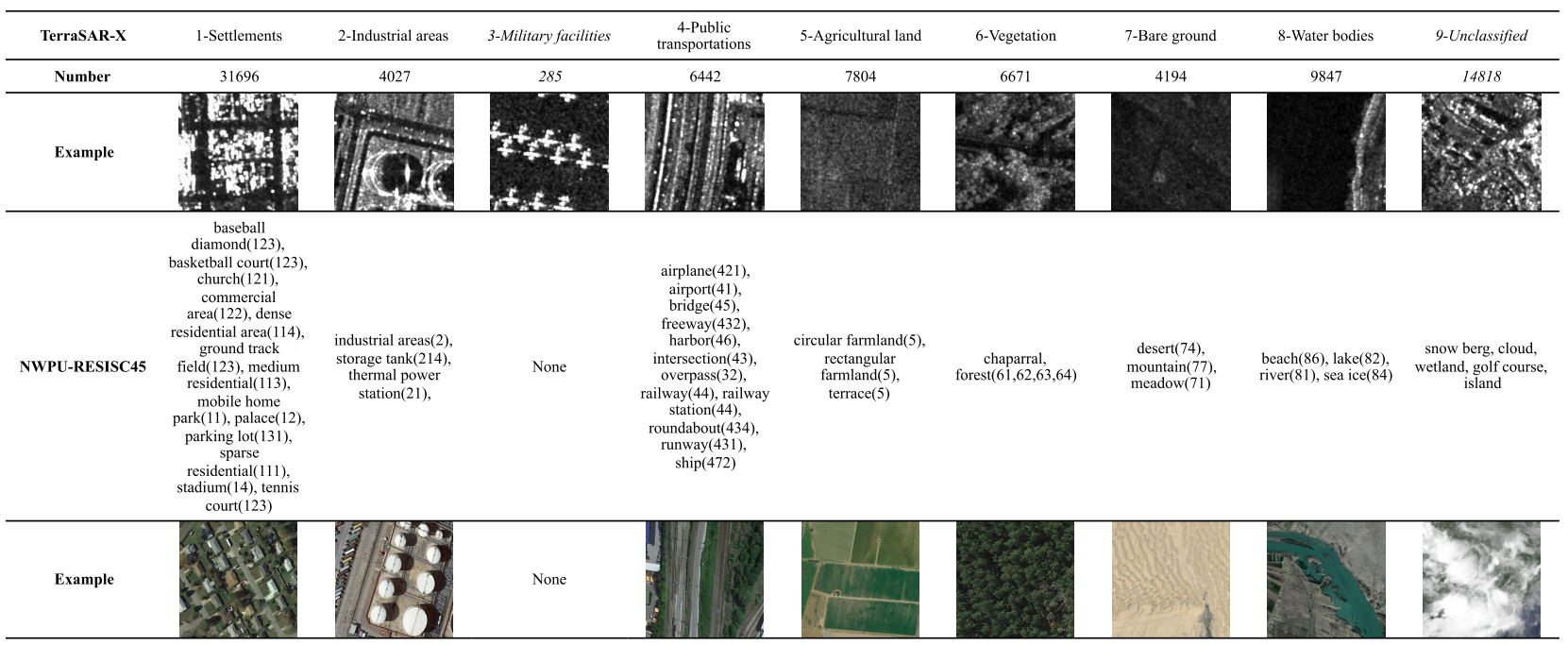

Fig. 1. Nine kinds of the land cover of our annotated level-1 TSX data set with imbalanced classes. Statistically, 40 categories in NWPU-RESISC45 are semantically similar to the TSX data set. The number in brackets indicates the assigned label of TSX, which can be indexed in [7, Appendix, Table A.I.]. Due to the small number of patches in "Military Facilities" and indistinct label "Unclassified," only seven classes are applied in our experiments.

\section{DATA SETS}

The HR SAR annotated land cover data set [7] exploited in this letter has been collected from the X-band TSX instrument [8]; we used horizontally polarized $(\mathrm{HH})$, multilook ground range detected (MGD) products that were radiometrically enhanced. The selected SAR images were taken in high-resolution spotlight mode with a resolution of $2.9 \mathrm{~m}$, acquired with an incidence angle between $20^{\circ}$ and $50^{\circ}$, in both descending and ascending pass directions.

This annotated semantic data set covers urban and nonurban areas from all over the world, grouped into 46 collections in total. With a pixel spacing of $1.25 \mathrm{~m}$, each SAR image was tiled into nonoverlapping patches with a size of $160 \times 160$ pixels, resulting in $200 \times 200 \mathrm{~m}^{2}$ on the ground so that a patch covered a typical object on the ground. A single label was assigned to each patch based on the dominating class. With three-level hierarchical annotation, only level-1 labels are used in this letter.

Automatically interpreting the worldwide large-scale SAR data set is challenging due to the following reasons.

1) Highly Imbalanced Classes: As shown in Fig. 1, the imbalanced classes result in a big difference in data volume for each category in level-1 annotation case, mainly due to the fact that the data set focuses on urban areas. This imbalance problem appears more serious in level-2 and level-3 annotations.

2) Geographic Diversity: Collected world-widely, different architectural styles of cities bring out a large geographic diversity of images. The typical regional characteristics result in high intraclass diversity and interclass similarity, as shown in Fig. 2. Besides, the acquisition period of these images ranges from 2007 to 2012. During this period of time, some nonconstant parameters, such as the radiometric calibration factor, will result in different characteristics of the SAR images.

3) Label Noise: By covering an area of $200 \times 200 \mathrm{~m}^{2}$ on the ground, sometimes, a patch contains more than one land cover type. Even though the annotation was based on the dominating
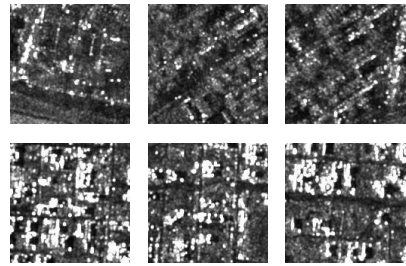

\section{Residential Areas} Level-1 label: Settlements

Upper: Calgary (Canada) Lower: Bayaa (Iraq)
Fig. 2. High intraclass diversity of "low-density residential areas" in two different cities.

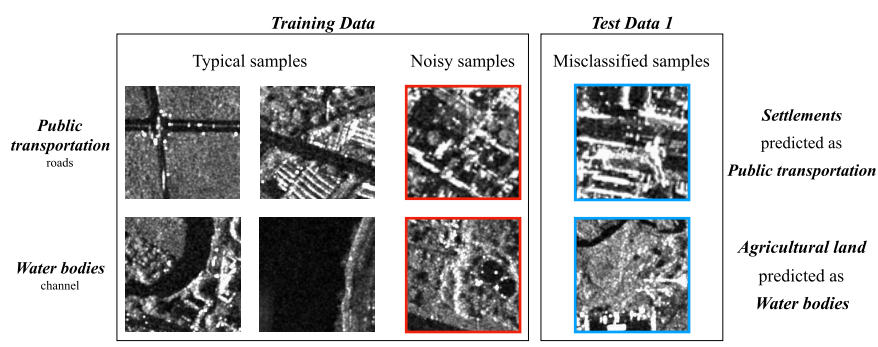

Fig. 3. Examples of label noise and model bias due to the noisy labels.

class, there still existed some incomplete annotations or some semantic biases [9], which may lead the trained model to overfitting when using these misleading samples. Fig. 3 gives an example of label noise in our data set.

Due to the low number of only 285 patches from two collections of "Military Facilities" and the unlabeled patches of "Unclassified", we only used the remaining seven land cover classes in this letter.

Another remote sensing LULC data set used in this letter is NWPU-RESISC45 [10], which has a land cover annotation similar to the seven classes of TSX data set used in our experiment, as shown in Fig. 1, containing 31500 images covering 45 scene classes.

\section{Proposed Method}

Facing the above-mentioned difficulties, we propose a deep transfer learning-based method in this part to tackle the SAR land cover classification issue. 


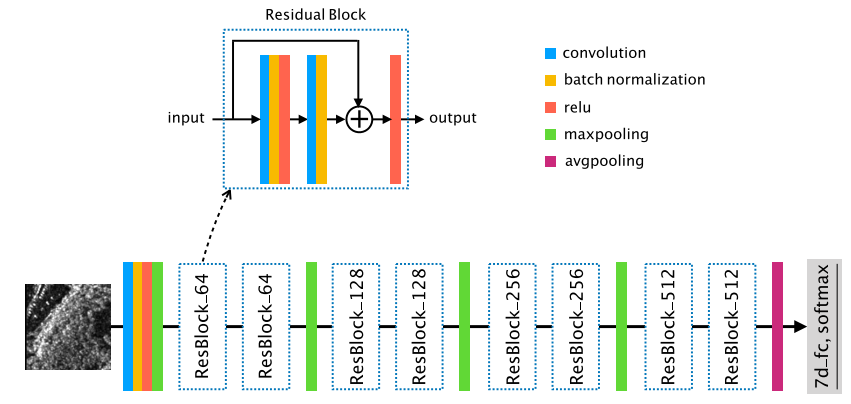

Fig. 4. Residual network architecture applied in our experiments.

\section{A. Transfer Learning With Deep Residual Networks}

ResNet-18 [11] shown in Fig. 4, containing four residual blocks repeated twice, with two convolution layers in each block, followed by an average pooling layer and a fully connected layer related to the class number of the task, was applied in our experiments. Such a very deep well-trained neural network (with a data volume of about 43.7 MB) has a great ability in extracting hierarchical features but is difficult to train from scratch. Transfer learning provides a better initialization to the parameters in deep layers, making the optimization easier and speeding up the training process. The ImageNet pretrained model is popular as a transferred source. However, the large difference lying between SAR land cover patches and natural object images may harm the transferability, making it necessary to find a bridge to narrow the gap [12].

Rather than using the ImageNet pretrained model as most remote sensing applications do, we proposed a transitive transfer learning method with the help of the NWPURESISC45 data set [10] to build a bridge linking the natural and SAR land cover images. ResNet-18 pretrained on ImageNet [11] was applied as our initial model, and then, the convolution layers were fine-tuned with the NWPURESISC45 data set, obtaining the remote sensing enhanced model as the transfer source for our applications.

\section{B. Imbalanced Classification}

When we trained a model with a data set of highly imbalanced classes, we applied some techniques, such as sampling and cost-sensitive learning [13]. For evaluation, we considered some metrics beyond accuracy, such as precision, recall, and F1-score.

Each time when we input a batch of samples to the network, oversampling of the minority classes and undersampling of the majority classes were applied to ensure a balanced distribution in that batch. Moreover, the training samples were weighted according to the corresponding proportions of the data set in order to penalize the misclassification of the minority classes more heavily than for the majority classes.

\section{Top-2 Smooth Loss Function}

Here, we introduced the top-2 smooth loss function [14] to reduce the label noise in our data set. With the classification of seven types of land cover in level-1, we mainly focused on the top- 2 predictions $\overline{\mathbf{y}} \in \mathbb{R}^{2}$. We define the label space
$\mathcal{Y}=\{1,2, \ldots, n\}$ and the ground truth label $y \in \mathcal{Y}$, the top2 predictions $\overline{\mathbf{y}} \in \mathcal{Y}^{(2)}$. The subset of $\mathcal{Y}^{(2)}$, where the ground truth $y$ is included, is written as $\mathcal{Y}_{y}^{(2)}$. The top-2 smooth loss function with temperature can be defined as

$$
\begin{array}{r}
L_{2, \tau}(\mathbf{s}, y)=\tau \log \left[\sum_{\overline{\mathbf{y}} \in \mathcal{Y}^{(2)}} \exp \left(\frac{1}{\tau}\left(\Delta(\overline{\mathbf{y}}, y)+\frac{1}{2} \sum_{j \in \overline{\mathbf{y}}} s_{j}\right)\right)\right] \\
-\tau \log \left[\sum_{\overline{\mathbf{y}} \in \mathcal{Y}_{y}^{(2)}} \exp \left(\frac{1}{2 \tau} \sum_{j \in \overline{\mathbf{y}}} s_{j}\right)\right]
\end{array}
$$

where $\mathbf{s} \in \mathbb{R}^{n}$ denotes a vector of scores of $n$ labels, $\tau$ is the temperature parameter, and $\Delta(\overline{\mathbf{y}}, y) \triangleq \mathbb{1}(y \notin \overline{\mathbf{y}})$ denotes the margin. The top- 2 smooth loss function takes two most likely predictions into consideration, making it possible to prevent overfitting with label noise when learning only with the cross-entropy loss of a single label.

In the proposed method, the top- 2 smooth loss was combined with the cross-entropy loss with a tradeoff $\lambda$ rather than totally replacing it as [14] did because $k=2$ is not much smaller than $n=7$ in our case. The top- 2 loss was used as a constraint to alleviate model bias toward the single misleading label when there was more than one kind of land cover on the ground. Finally, adding an L2 regularization term and the loss weight $w_{y}$, the combined loss function can be written as

$$
\begin{aligned}
L_{2, \tau, \mu, \lambda}(\mathbf{s}, y)=(1-\lambda) & \left(-\sum_{i} s_{i} \log \frac{\exp \left(s_{i}\right)}{\sum_{j=1}^{C} \exp \left(s_{j}\right)}\right) \\
& +w_{y} \lambda L_{2, \tau}(\mathbf{s}, y)+\mu \sum_{i}\left\|\beta_{i}\right\|^{2}
\end{aligned}
$$

where $\beta_{i}$ denotes the weights in each layer and $\lambda, \tau$, and $\mu$ are three hyperparameters that control the tradeoff between the top-2 and cross-entropy losses, temperature tuning, and the penalty of the regularization term. The cost weight $w_{y}$ related to the class $y$ is given by

$$
w_{y}=\frac{1}{n-1}\left(1-\frac{N_{y}}{\sum_{i=1}^{n} N_{i}}\right)
$$

where $n$ denotes the class number and $N_{i}$ denotes the number of samples in the $i$ th class.

In addition, we use bagging [15], training five submodels with resampling data, and voting the predictions of all submodels in order to make the trained model more robust and general.

\section{EXPERIMENTAl Results AND Discussion}

We first introduce our experimental settings in Section IV-A. In Sections IV-B and IV-C, we analyze the effectiveness of different transfer sources and the combined top-2 smooth loss. Finally, we use three examples to demonstrate the good generalization of the proposed SAR specific deep model in IV-D.

\section{A. Experimental Settings}

On the one hand, we randomly chose 200 patches in each category from collection 01 to 36 as Test Data 1 and the 
TABLE I

TRAINING AND TEST DATA SET

\begin{tabular}{ccccccccc}
\hline Category & Settlements & $\begin{array}{c}\text { Industrial } \\
\text { areas }\end{array}$ & $\begin{array}{c}\text { Public } \\
\text { transportations }\end{array}$ & $\begin{array}{c}\text { Agricultural } \\
\text { land }\end{array}$ & $\begin{array}{c}\text { Natural } \\
\text { vegetation }\end{array}$ & $\begin{array}{c}\text { Bare } \\
\text { ground }\end{array}$ & $\begin{array}{c}\text { Water } \\
\text { bodies }\end{array}$ & Total \\
\hline Training Data & 24,930 & 2,979 & 4,485 & 6,029 & 4,911 & 2,240 & 6,826 & $\mathbf{5 2 , 4 0 0}$ \\
Test Data 1 & 200 & 200 & 200 & 200 & 200 & 200 & 200 & $\mathbf{1 , 4 0 0}$ \\
Test Data 2 & 3,155 & 563 & 928 & 925 & 1,383 & 1,319 & 2,133 & $\mathbf{1 0 , 4 0 6}$ \\
\hline
\end{tabular}

remaining as Training Data. On the other hand, patches of the full scene images from another seven collections were used as Test Data 2 that had a different distribution with Training Data and Test Data 1. The details of training and test data settings are presented in Table I.

The TSX detected images are represented by 16 bits spanning a large dynamic range. In our experiments, the 16-bit data after the log transform was fed into the network to prevent the gradients from vanishing during training. When transferring the pretrained model, only the last fully connected layer was adapted to fit the new task.

The hyperparameters of $\lambda, \tau$, and $\mu$ that control the tradeoff between the top- 2 and cross-entropy losses, the temperature tuning, and the penalty of the regularization term were empirically set to $0.2,1.0$, and 0.25 , respectively. The Adam optimization algorithm [16] was applied, and the initial learning rate was set to $10^{-5}$ when using the top- 2 loss and to $10^{-4}$ otherwise.

\section{B. Transitive Transferring Model}

We compared the results of overall classification accuracy in Test Data 1 and the average stopping time of training a ResNet-18 network for the following cases to demonstrate the effectiveness of transferring from proper source data to SAR land cover classification.

1) RD-TSX: By randomly initializing all layers of ResNet-18, the network was trained with the TSX data set from scratch.

2) TL-ImageNet2TSX: By using the pretrained model of ImageNet, the network was fine-tuned with the TSX data set with only the last layer related to the classes trained from scratch.

3) TL-ImageNet2RS2TSX: The hierarchical convolution layers of ImageNet pretrained model were fine-tuned with NWPU-RESISC45 optical remote sensing images for 45 types of land cover classification and then transferred to the TSX data set.

Fig. 5 illustrates that training such a very deep network, such as ResNet-18, with the TSX data set from scratch is difficult and time-consuming. Consequently, it is necessary to find a better initialization of millions of parameters. Although the ImageNet pretrained model has been widely used for remote sensing applications, it is controversial to directly transfer the natural images to SAR data due to the huge differences between them. Here, we took advantage of the remote sensing images with similar categories to our SAR land cover data set to narrow the gap between natural images and SAR data. We see a performance improvement both in test accuracy and training time.

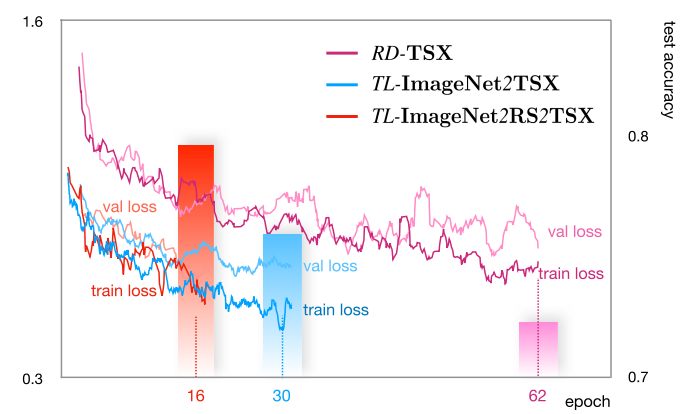

Fig. 5. Lines denote the loss values on training and validation data before early stopping, and the bars denote the validation accuracy of three experimental settings.

TABLE II

Classification Result on Test Data 1 and Test Data 2

\begin{tabular}{ccccc}
\hline & Eval & ce loss & top-2 loss & combined loss \\
\hline \multirow{3}{*}{ Test Data 1 } & F1 score & 0.8318 & 0.7954 & $\mathbf{0 . 8 3 2 7}$ \\
& top-1 acc & $83.14 \%$ & $79.5 \%$ & $\mathbf{8 3 . 2 1 \%}$ \\
& top-2 acc & $\mathbf{9 5 . 3 6 \%}$ & $93.29 \%$ & $95.00 \%$ \\
\hline \multirow{2}{*}{ Test Data 2 } & F1 score & 0.709 & 0.684 & $\mathbf{0 . 7 2}$ \\
& top-1 acc & $74.09 \%$ & $73.24 \%$ & $\mathbf{7 6 . 7 8 \%}$ \\
& top-2 acc & $91.26 \%$ & $91.25 \%$ & $\mathbf{9 2 . 6 2 \%}$ \\
\hline \multicolumn{2}{c}{ training time } & 16 epochs & 6 epochs & 9 epochs \\
\hline
\end{tabular}

\section{Combined With Top-2 Loss}

Besides applying some strategies, such as Dropout and L2 regularization, to avoid overfitting during training, we found that the label noise may play an important role with a negative influence on the trained model. To address this prediction bias issue, we introduced a top- 2 smooth loss function to constrain the cross-entropy from introducing a bias to the noisy labels. We use different metrics of top-1, top-2 overall accuracy, and also F1-score to evaluate the results, which is defined by $F_{1}=2 /\left(p^{-1}+r^{-1}\right)$, where $p=(\mathrm{TP} /(\mathrm{TP}+\mathrm{FP})), r=(\mathrm{TP} /(\mathrm{TP}+\mathrm{FN}))$, and $\mathrm{TP}, \mathrm{FP}$, and FN denote the true positive, false positive, and false negative samples, respectively.

The top-2 smooth loss focuses on two most likely land cover classes, thus penalizing the predictions only when neither of the top-2 likely labels is the ground truth. Table II shows the training time of using different loss functions and the test results of F1-score, top-1, and top-2 accuracies both on Test Data 1 and Test Data 2. The top-2 accuracy is the percentage of time that the classifier gives the correct class in the top-2 probabilities. We found that training with top-2 smooth loss only would lead to fast convergence of the deep network to speed up training. However, the top-2 smooth loss was too relaxing for the optimization resulting in much smaller 


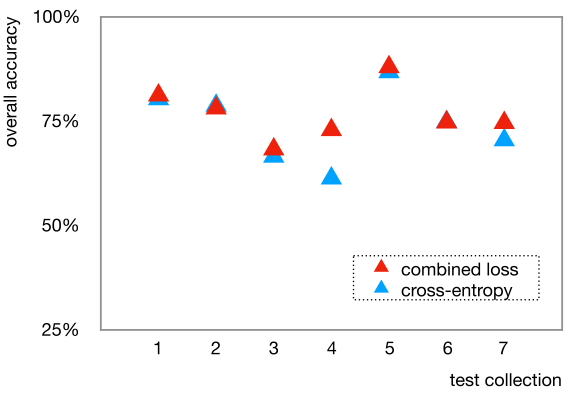

Fig. 6. Classification results on seven new collections of Test Data 2, learning with cross-entropy, and the combined loss shown as blue/red dots, respectively.

TABLE III

TRANSFER TO NEW TASKS ON SAR DATA

\begin{tabular}{cccc}
\hline Dataset & Task & Classes & Transfer Result \\
\hline Test Data 2 & scene classification & 7 & $76.78 \%$ \\
MSTAR [17] & target recognition & 10 & $99.46 \%$ \\
\hline
\end{tabular}

gradients after a period of time and weights being difficult to update. As a consequence, we combined the top-2 smooth loss with the cross-entropy as a constraint to achieve a faster convergence with nine epochs compared with 16 epochs of learning with cross-entropy. With the improvement in F1-score and accuracy, together with the obvious speedup in training time, the combined loss performed considerably better.

\section{Generalization to Other Data and Tasks}

We could demonstrate the good generalization of our SAR deep ResNet model by testing two tasks: 1) land cover classification on new collections of Test Data 2 and 2) MSTAR [17] target recognition without data augmentation, as shown in Table III.

Fig. 6 shows the test results of each new collection in Test Data 2 that has a different distribution with the training data. In most new collections, the performance of the combined loss was better than cross-entropy (2.69\% higher on average), which demonstrates that the trained model with combined loss reduces overfitting due to label noise and improves generalization on new data set.

Furthermore, when we transferred the proposed model to an MSTAR [17] 10-class recognition task, we achieved a stateof-the-art overall accuracy of $99.46 \%$, compared with $98.02 \%$ using an ImageNet pretrained model, and $99.09 \%$ by other CNN-based methods [18].

\section{CONCLUSION}

In this letter, a deep transfer learning method combining the top- 2 smooth loss was proposed to solve the land cover classification problem for a large-scale HR TSX data set, with highly imbalanced classes, geographic diversity, and noisy labels. Rather than applying the ImageNet pretrained model of ResNet-18 to SAR images directly, we used an optical remote sensing land cover data set to narrow the gap between SAR and natural images, which results in a significant improvement in feature transferability. The proposed combined loss function is successful in reducing model bias to noisy labels, and our proposed deep ResNet model shows a good generalization, which allows it to be exploited for other SAR-specific tasks.

\section{ACKNOWLEDGMENT}

The authors would like to thank the TSX Science Service System for the provision of images (Proposals MTH-1118 and LAN-3156). They would also like to thank Dr. G. Schwarz for improving this letter.

\section{REFERENCES}

[1] Q. Lv, Y. Dou, X. Niu, J. Xu, J. Xu, and F. Xia, "Urban land use and land cover classification using remotely sensed SAR data through deep belief networks," J. Sensors, vol. 2015, pp. 1-10, 2015.

[2] L. Zhang, W. Ma, and D. Zhang, "Stacked sparse autoencoder in PolSAR data classification using local spatial information," IEEE Geosci. Remote Sens. Lett., vol. 13, no. 9, pp. 1359-1363, Sep. 2016.

[3] Z. Huang, Z. Pan, and B. Lei, "Transfer learning with deep convolutional neural network for SAR target classification with limited labeled data," Remote Sens., vol. 9, no. 9, p. 907, Aug. 2017.

[4] W. Wu, H. Li, L. Zhang, X. Li, and H. Guo, "High-resolution PolSAR scene classification with pretrained deep convnets and manifold polarimetric parameters," IEEE Trans. Geosci. Remote Sens., vol. 56, no. 10, pp. 6159-6168, Oct. 2018.

[5] C. Hütt, W. Koppe, Y. Miao, and G. Bareth, "Best accuracy land use/land cover (LULC) classification to derive crop types using multitemporal, multisensor, and multi-polarization SAR satellite images," Remote Sens., vol. 8, no. 8, p. 684, Aug. 2016.

[6] J. Zhao, W. Guo, B. Liu, Z. Zhang, W. Yu, and S. Cui, "Preliminary exploration of SAR image land cover classification with noisy labels," in Proc. IEEE Int. Geosci. Remote Sens. Symp. (IGARSS), Jul. 2017, pp. 3274-3277.

[7] C. O. Dumitru, G. Schwarz, and M. Datcu, "Land cover semantic annotation derived from high-resolution SAR images," IEEE J. Sel. Topics Appl. Earth Observ. Remote Sens., vol. 9, no. 6, pp. 2215-2232, Jun. 2016.

[8] TerraSAR-X Basic Product Specification Document, Issue 1.9. Accessed: Dec. 10, 2018. [Online]. Available: https://sss.terrasar-x.dlr.de/docs/TXGS-DD-3302.pdf

[9] A. Murillo Montes De Oca, R. Bahmanyar, N. Nistor, and M. Datcu, "Earth observation image semantic bias: A collaborative user annotation approach," IEEE J. Sel. Topics Appl. Earth Observ. Remote Sens., vol. 10, no. 6, pp. 2462-2477, Jun. 2017.

[10] G. Cheng, J. Han, and X. Lu, "Remote sensing image scene classification: Benchmark and state of the art," Proc. IEEE, vol. 105, no. 10 pp. 1865-1883, Oct. 2017.

[11] K. He, X. Zhang, S. Ren, and J. Sun, "Deep residual learning for image recognition," in Proc. IEEE Conf. Comput. Vis. Pattern Recognit. (CVPR), Jun. 2016.

[12] Z. Huang, Z. Pan, and B. Lei, "What, where, and how to transfer in SAR target recognition based on deep CNNs," IEEE Trans. Geosci. Remote Sens., pp. 1-13, 2019.

[13] M. Kukar and I. Kononenko, "Cost-sensitive learning with neural networks," in Proc. ECAI, 1998, pp. 445-449.

[14] L. Berrada, A. Zisserman, and M. P. Kumar, "Smooth loss functions for deep top-k classification," in Proc. IEEE Int. Conf. Learn. Represent. (ICLR), 2018, pp. 1-25.

[15] E. Bauer and R. Kohavi, "An empirical comparison of voting classification algorithms: Bagging, boosting, and variants," Mach. Learn., vol. 36, nos. 1-2, pp. 105-139, 1999.

[16] D. P. Kingma and J. Ba, "Adam: A method for stochastic optimization," in Proc. 3rd Int. Conf. Learn. Represent. (ICLR), 2015, pp. $1-15$.

[17] The Air Force Moving and Stationary Target Recognition Database. Accessed: Feb. 3, 2016. [Online]. Available: https://www.sdms. afrl.af.mil/datasets/mstar/

[18] S. Chen, H. Wang, F. Xu, and Y.-Q. Jin, "Target classification using the deep convolutional networks for SAR images," IEEE Trans. Geosci. Remote Sens., vol. 54, no. 8, pp. 4806-4817, Aug. 2016. 